\title{
Quando o parceiro sem fim lucrativo falha? Uma analise das PNPs na área de saúde no Rio de Janeiro
}

\author{
Alketa Peci, Aline Santos y Desiree Machado \\ Fundaçao Getulio Vargas
}

\section{Resumo}

O objetivo desta pesquisa é a capacidade de associação entre associações sem fins lucrativos e instituições públicas. Para este propósito, investigamos treze organizações, pertencentes às Organizações Sociais de Saúde (OSS), com o governo da cidade do Rio de Janeiro. Foi construída uma tipologia para associações, que varia de acordo com a capacidade organizacional. A análise indica que há três resultados da colaboração: sinergia, complementaridade e implementação. Os achados mostram que o OSS estudado mostra um alto grau de capacidade organizacional e sinergia.

Palavras-chave: Capacidade organizacional, organizações públicas sem fins lucrativos, de cuidados de saúde primários, metodologias indutivas.

When does the non-profit partner fail? An analysis of the PNPs in the health area in Rio de Janeiro

\begin{abstract}
The aim of this research is the capacity of association between non profit-making associations and public institutions. To this objetive, we investigate thirteen organizations, belonging to the Organizações Sociais de Saúde (OSS), with the government of the city of Rio de Janeiro. A typology for associations was constructed, which varies according to organizational capacity. The analysis indicates that there are three collaboration's outcomes: synergy, complementarity and implementation. The findings show that the OSS studied show a high degree of organizational capacity and synergy.
\end{abstract}

Keywords: Organizational capacity, Public-Nonprofit Partnership, primary health care, inductive methodologies.

*Dirección de correspondencia [Correspondence address]: Alketa Peci, Aline Santos y Desiree Machado, Fundaçao Getulio Vargas

E-mail: Alketa.Peci@fgv.br 


\section{Introdução}

O número de parcerias entre o setor público com organizações sem fins lucrativos (public-nonprofit partnerships - PNPs) vem crescendo de modo considerável em todo o mundo. De forma geral, fomentadas pelo parceiro público, as PNPs são geralmente justificadas pelas várias dificuldades gerenciais enfrentadas pelo governo com a expectativa de que parceiros sem fins lucrativos poderão fornecer melhores resultados na entrega dos serviços públicos. No Brasil, essa importante forma de colaboração está presente em importantes áreas de políticas públicas, como a saúde, cultura ou ciência e tecnologia.

No entanto, o modelo de PNPs tem sido confrontado por denúncias de corrupção, influenciando negativamente a opinião pública. Baseado na premissa de que a PNP é afetada pela capacidade da organização sem fim lucrativo de gerir e entregar serviços públicos, pesquisamos esse tipo de colaboração por um ângulo pouco estudado: nosso objetivo é medir a capacidade da parceria, focando na combinação das capacidades organizacionais dos parceiros (sem fins lucrativos e público) em alcançar objetivos mais ambiciosos de políticas públicas.

Especificamente, nossa pesquisa incidiu sobre as 13 (treze) Organizações Sociais de Saúde (OSS) que integram as PNPs com a prefeitura da cidade do Rio de Janeiro. A partir da análise do banco de dados da Secretaria Municipal de Saúde, criamos uma tipologia para as parcerias que varia de acordo com a capacidade organizacional: os parceiros "públicos", os subcontratados e os oportunistas. Nossa análise considerou várias dimensões organizacionais e indicou o surgimento de três tipos de resultados que surgem de cada tipo de colaboração: sinergia, complementaridade e imposição.

Nossos resultados apresentam conclusões sobre as OSS "públicas" que demonstraram elevados graus de capacidade organizacional e sinergia: na verdade, a origem dessas organizações sem fins lucrativos está imbricada a própria história de organizações do setor público. Interpretamos tais achados com base nas especificidades do contexto histórico brasileiro.

\section{Capacidade Organizacional}

Entre os vários argumentos para as PNPs, prevalecem os conceitos de complementaridade e de sinergia (Skelcher, 2005). As PNPs são geralmente justificadas pelas inúmeras dificuldades gerenciais enfrentadas pelo setor público, além da expectativa de que as organizações sem fins lucrativos proporcionem melhores resultados na entrega dos serviços públicos. Com base na premissa de que nem o setor público nem o terceiro setor são capazes de resolver problemas sociais complexos por conta própria, espera-se que as parcerias permitam que diferentes pessoas e organizações se combinem e capitalizem pontos fortes e capacidades complementares (Reich, 2002; Lasker et al., 2001: 180).

Como resultado, a expectativa é de que as parcerias reduzam custos, melhorem a qualidade e maximizem a eficiência (Alexander y Nank, 2009; Lambright et al., 2010; Lecy y Van Slyke, 2013). Tais melhorias dependem de uma premissa implícita que os parceiros sem fins lucrativos contam com uma melhor capacidade para prestar os serviços públicos.

O conceito de capacidade no que tange às parcerias é problemático visto que, correntemente, aplicam-se às dimensões organizacionais de um único parceiro. De fato, a maior parte da literatura adapta as dimensões relacionadas com o conceito de capacidade organizacional, geralmente definido em termos de atributos organizacionais que ajudam ou permitem que uma única organização cumpra sua missão (Balduck et al., 2015; Eisinger, 2002; Mcphee y Bare, 2001). Entretanto, este conceito centra-se sobre o desempenho de uma única organização, ao invés do desempenho da parceria.

Por outro lado, a noção de capacidade colaborativa emerge como alternativa importante para essa abordagem organizacional única e, simultaneamente, como um complexo conceito multinível. A capacidade de colaboração refere-se às condições necessárias para que as coalizões promovam uma colaboração eficaz e construam mudanças sustentáveis (Goodman et al., 1998; Foster-Fishman et al., 2001). Parcerias contraproducentes podem levar à desconfiança e ao pessimismo, corroendo as bases para futuras colaborações (Alexander et al., 2003; Huxham y Vangen, 2000; Alexander y Weiner, 1998).

A questão central de uma colaboração eficaz reside na relação entre a capacidade organizacional de cada parceiro e a capacidade de colaboração da parceria. Goodman et al. (1998) partilham a ideia de que a capacidade de colaboração é uma condição necessária para o desenvolvimento, implementação e manutenção de uma parceria de promoção à saúde e prevenção de doenças, por exemplo.

Tal visão mais ampla, baseada na sustentabilidade das parcerias, indica a necessidade de verificação da capacidade colaborativa em quatro níveis críticos: (a) dos membros de organizações parcei- 
ras; (b) de suas relações; (c) de sua estrutura organizacional; e (d) dos programas financiados.

Nosso foco nesta pesquisa reside na capacidade colaborativa das parcerias no nível organizacional, com o objetivo de aproximar ambas as perspectivas supramencionadas, assumindo que a cooperação organizacional é uma condição estrutural e necessária (embora não suficiente) para a sustentabilidade da parceria. Em nosso trabalho de campo investigamos um tipo de parceria que direciona esse foco conceitual e empírico da pesquisa. Isso porque o contexto das parcerias brasileiras na área da saúde é caracterizado por uma abordagem contratual e altamente formalista em relação às parcerias.

Nesse âmbito, a capacidade pode ser amplamente definida como o conjunto de esforços para gerar os produtos e os resultados desejados (Balduck et al., 2015) e é explicitamente relacionada com a expectativa da sinergia organizacional ou da complementaridade de ambos os parceiros. As vantagens das parcerias ocorrem quando os parceiros podem combinar seus recursos humanos e materiais para a consecução de objetivos ambiciosos, os quais não seriam atingidos sem a parceria (Kanter, 1994; Lasker, 1997; Mayo, 1997; Butterfoss et al., 1996). No entanto, a complementaridade e a sinergia são tratadas como resultados semelhantes que emergem de acordos de colaboração. Defende-se ainda que a sinergia, quando a colaboração cria algo maior que a soma das partes individuais, é o único resultado desejável de uma parceria vantajosa (Lasker et al., 2001).

\section{Dimensões da capacidade organizacio- nal que contribuem para as parcerias}

A literatura sobre as PNPs, embora muito vasta em termos de cooperação, geralmente tem negligenciado o papel específico das organizações sem fins lucrativos na promoção e manutenção das parcerias. De acordo com Paynter y Berner (2014), os estudos sobre capacidade organizacional não abrangem seu impacto na prestação do serviço. Existe a crença de que, sempre que a PNP é estabelecida, o parceiro público confia que a organização sem fins lucrativos possui um nível adequado para entregar os serviços (Fredericksen y London, 2000).

$\mathrm{Na}$ prática, a capacidade organizacional do parceiro público e da organização sem fins lucrativos emerge como uma dimensão significativa para o sucesso da PNP, sobretudo porque é um conceito multidimensional, pois destaca o papel de elementos específicos que podem contribuir com os resultados da parceria como um todo. Assim, a sinergia ou complementaridade esperada das parcerias de- rivam da combinação das dimensões da capacidade organizacional do parceiro público e da organização sem fins lucrativos.

A capacidade organizacional surgiu na literatura sem fins lucrativos com um referencial teórico significativo, fornecendo uma base para a análise holística dos fatores que contribuem para a realização dos objetivos da organização e, mais amplamente, para a eficácia organizacional (Eisinger, 2002; Millar y Doherty, 2016). Em outras palavras, destaca-se a habilidade das organizações em mobilizar os recursos ou capitais em direção ao cumprimento da missão orgazanicional (Balduck et al., 2015; Eisinger, 2002; Hall et al., 2003; Sharpe, 2006). A pesquisa é dispersa no que tange aos conceitos alternativos da capacidade organizacional, como capacidade de gestão (Sowa et al., 2004) ou capacidade administrativa (Lodge y Wegrich, 2014), contudo é rica em termos das dimensões específicas relacionadas à capacidade do desempenho organizacional.

Adicione-se ao fato de que a capacidade organizacional caracteriza-se por ser um conceito multidimensional, já que inclui uma série de atributos organizacionais que são considerados significativos para a organização atingir suas metas. De fato, a capacidade remete ao modus operandi, à estrutura, ao processo e ao envolvimento dos membros da organização (Sowa et al., 2004).

Traduzindo esse conceito, a capacidade é constituida de três dimensões-chave de capital: humano, estrutural e financeiro (Sharpe, 2006). Hall et al. (2003) selecionam cinco dimensões: a) recursos humanos; b) finanças; c) relacionamentos e redes; d) infraestrutura; e e) planejamento e desenvolvimento. Já Misener y Doherty (2009) focam em recursos humanos, recursos financeiros, formalização e ligações externas. Para Eisinger (2002), liderança eficaz, pessoal qualificado e suficiente, algum nível de institucionalização e relacionamento com a comunidade podem compor influências para a capacidade organizacional. Em seu estudo sobre o desenvolvimento da capacidade organizacional, Fredericksen y London (2000) destacam quatro dimensões: a) liderança e visão; b) gestão e planejamento; c) planejamento e prática fiscal; e d) suporte operacional.

Naturalmente, as dimensões relacionadas com a capacidade organizacional dependem do tipo de parceria. As ligações externas, embora importantes para os organismos comunitários, não são muito relevantes para as parcerias estritamente formais existentes no Brasil. Já outras dimensões são particularmente relevantes porque podem contribuir para a complementaridade ou a sinergia em uma lógica 
de parceria.

Em nossa análise nos concentramos nas seguintes dimensões: 1- liderança e visão; 2- grau de institucionalização dos processos e rotinas organizacionais; 3- principais fontes de financiamento (planejamento e práticas Fiscais); e 4- apoio operacional (inclusive pessoal qualificado e suficiente, espaço físico e equipamentos).

\section{Liderança e visão}

Segundo Fredericksen y London (2000), esta dimensão inclui a presença de um conselho executivo coeso que articula a gestão por meio da declaração de visão/missão. A liderança é amplamente investigada na literatura sem fins lucrativos como uma dimensão indispensável da capacidade organizacional (Fredericksen y London, 2000; Henton et al., 1997; Heimovics et al., 1993). A liderança eficaz é caracterizada pelo compromisso com a missão, solidez e metas eficazes (Rainey y Steinbauer, 1999). Nos concentramos em duas dimensões principais: as declarações de missão e a composição dos conselhos executivos.

A declaração de missão deve descrever, de forma sucinta, o porquê da existência da organização. É primordial que a missão seja compartilhada com todos seus stakeholders, viabilizando o entendimento de todos os envolvidos na parceria. A descrição pública da missão também proporciona controle e transparência para a sociedade (Brasil, 1997), justificando a própria celebração das parcerias.

Em última análise, todas as dimensões da capacidade organizacional estão relacionadas ao cumprimento da missão (Rainey y Steinbauer, 1999; Paynter y Berner, 2014). Ainda que as declarações de missão sejam relevantes, consideramos a coerência da missão organizacional quando a parceria foi constituída, investigando como ocorre a combinação das missões dos parceiros públicos e sem fins lucrativos em busca de objetivos mais ambiciosos.

Diferentes estruturas organizacionais, particularmente no papel de liderança, podem influenciar a capacidade da organização: um conselho constituído por profissionais remunerados podem gerir algumas organizações, enquanto comitês de voluntários lideram outras. Um aspecto relevante pode determinar uma organização de sucesso: se a diretoria executiva é remunerada (Paynter y Berner, 2014). Ademais, organizações que possuem uma diretoria executiva experiente são mais prováveis de serem equipadas com a expertise necessária para atuar nos cenários interno e externo, prosperando assim na flexibilidade e no desempenho alcançado (Mumford et al., 2000).

\section{Grau de institucionalização dos processos e rotinas organizacionais}

Está relacionado com o grau de estabilidade e de eficiência dos processos operacionais internos da organização (Zucker, 1987). Esses processos formais dão às partes interessadas os instrumentos para medir as forças, fraquezas ou necessidades. Também se refere aos planos e aos sistemas de apoio necessários para permitir o planejamento (orçamento, modelos de planejamento, informações sobre experiências organizacionais). Se a administração nem planeja nem possui os sistemas de apoio necessários para viabilizar o planejamento, a questão da capacidade é discutível (Paynter y Berner, 2014). Por fim, a capacidade de desenvolver e elaborar planos estratégicos organizacionais, planos de programas, políticas e propostas está incluída nos estudos de Hall, mas também na visão de Walker y Weinheimer (1998).

\section{Principais fontes de financiamento (Planeja- mento e Práticas Fiscais)}

Representa a capacidade de uma organização para captar e desenvolver recursos financeiros, e mais importante para parcerias, para contar com outras formas de financiamento. Hall et al. (2003) citam que as questões financeiras representam um óbice para as organizações no cumprimento de suas missões devido aos problemas associados ao financiamento de projetos e à falta de apoio financeiro para infraestrutura. Gumulka et al.(2005) relatam que as organizações desportivas e recreativas têm menos recursos financeiros do que muitos outros tipos de organizações sem fins lucrativos, resultando em uma variedade de problemas relacionados com a gestão financeira e com a aquisição de financiamento externo. Fredericksen y London (2000) argumentam que até a organização mais bem organizado é limitada sem recursos financeiros adequados e previsíveis para apoiar suas operações. Os autores estudaram se as fontes primárias e a previsibilidade dos fundos são autogeradas, subvenções públicas/privadas ou captação de fundos local. Graus de maturidade financeira, tais como o orçamento e a diversidade de financiamento, permitem que as organizações ajam deliberadamente em busca da missão e não em uma posição ad hoc, financeiramente vulnerável (Greenlee e Trussel, 2006; Schuh y Leviton, 2006). Organizações mais autônomas podem ser menos dependentes de financiadores isolados, aumentando seus recursos 
e se engajando mais prontamente em processos de planejamento estratégico (Vinzant y Vinzant, 1996).

\section{Apoio operacional}

É a capacidade de uma organização para implantar ou confiar em elementos organizacionais relacionados com as operações do dia-a-dia. O pessoal, a estrutura organizacional e as instalações afetam a capacidade organizacional para uma ação efetiva (Fredericksen y London, 2000). Nós nos concentramos em três dimensões importantes: a) pessoal qualificado; b) espaço físico; e c) equipamentos.

- Pessoal qualificado: dimensão definida como a capacidade do capital humano dentro da organização, também inclui as competências, conhecimentos, atitudes, motivação e comportamentos dos indivíduos da organização. Certamente, é a dimensão-chave que tem um impacto direto em todas as outras capacidades (Hall et al., 2003). Essa dimensão tem sido estudada e considerada como muito relevante na capacidade da comunidade (Chaskin, 2001) ou em organizações desportivas voluntárias (Adams y Deane, 2009; Cuskelly et al., 2004; Cuskelly et al., 2006; Kim et al., 2007; Seippel, 2002; Vos et al., 2012; Warner et al., 2011). Paynter y Berner (2014) argumentam que a capacidade organizacional pode ser facilmente ligada à gestão de recursos humanos, uma vez que atrair e reter pessoal suficiente, remunerado ou voluntário, é fundamental para a prestação de bons serviços. Fredericksen y London (2000) também defendem que o tamanho da organização, em termos de níveis de pessoal remunerado versus níveis de voluntariado, é fundamental para a sustentabilidade da aprendizagem organizacional e para a consistência das operações. Do mesmo modo, os autores avaliam a capacidade da organização para atrair e manter pessoal qualificado e voluntários.

- Espaço físico: normalmente, a infraestrutura organizacional também inclui recursos não pessoais que, na sua presença ou ausência, contribuem para a capacidade organizacional. Wicker y Breuer (2011) usaram a teoria da dependência de recursos e o conceito de capacidade organizacional para entender a escassez de recursos em clubes desportivos sem fins lucrativos alemães. O espaço físico acessível e adequado para as operaçôes contribui para a capacidade operacional de uma organização (Fredericksen y London, 2000).
- Equipamentos: a coordenação e o planejamento são muitas vezes necessários para garantir a provisão dos equipamentos (Poncelet y de Ville, 1995). Sistemas de garantia de qualidade podem ser necessários para determinar se uma organização está trabalhando de forma otimizada ou para ajudá-la a aprender e a melhorar (Muller, 1996). Na visão de Fredericksen y London (2000), a presença de equipamentos de suporte colabora com a capacidade organizacional.

\section{Metodologia}

Nossa pesquisa foi baseada em uma abordagem indutiva, visando construir uma tipologia de PNPs no setor de atenção primária à saúde (APS). Nossa pesquisa de campo focou em 13 (treze) Organizações de Saúde Social (OSS), os parceiros sem fins lucrativos, e a Secretaria Municipal de Saúde (SMS), o parceiro público, no Rio de Janeiro, de agosto de 2015 a junho de 2016.

É importante ressaltar que a cidade do Rio de Janeiro tem a maior concentração hospitalar municipal do Brasil. Isso se deve ao fato de que a cidade foi a capital brasileira no período compreendido entre 1763 e 1961. Essa peculiaridade levou o município a seguir sua própria lógica de regionalização: em 1999, 28 (vinte e oito) hospitais, clínicas e maternidades eram municipais (Oliveira, 2015). Em 2004, a cidade do Rio de Janeiro enfrentou uma grave crise no setor de saúde por conta das seguintes razões: a) baixa capacidade de investimento; b) excessivo gasto por conta do isolamento pessoal e político de outras esferas de governo e do setor privado; e c) custo extremamente alto da dívida municipal, resultando na incapacidade de assumir novos empréstimos.

De acordo com Mattos (2012), essa crise iniciou a discussão de um novo modelo de gestão da saúde no município. Após a promulgação da Lei $\mathrm{n}^{\mathrm{O}}$ 5.026, de 19 de maio de 2009, o prefeito Eduardo Paes implementou um novo modelo visando melhorar os procedimentos de contratação, reduzir custos e aumentar a transparência. Dessa forma, o município teve a possibilidade de estabelecer PNPs para aumentar a eficiência, flexibilizar relações trabalhistas e substituir contratos precários.

Destarte, o Poder Executivo pôde qualificar como Organizações Sociais as pessoas jurídicas de direito privado, sem fins lucrativos, cujas atividades sejam dirigidas ao ensino, à pesquisa científica, ao desenvolvimento tecnológico, à proteção e preservação do ambiente, à cultura, à saúde e ao esporte, atendidos 
aos requisitos da Lei $n^{\underline{0}} 5.026 / 2009$.

São consideradas Organizações Sociais no âmbito da Prefeitura do Rio de Janeiro, para efeitos da Lei $\mathrm{n}^{\mathrm{O}} 5.026 / 2009$, as entidades cujos pedidos de qualificação foram deferidos pela Comissão de Qualificação (COQUALI), estando aptas a assinar contrato de gestão com a Administração Municipal na área de atuação que foram qualificadas. Entende-se por contrato de gestão o instrumento firmado entre o Poder Público e a entidade qualificada como Organização Social, com vistas à formação de parceria entre as partes para fomento e execução de atividades relativas às áreas previstas em lei. Para qualificar-se, a Organização Social deve ter finalidade não-lucrativa, com a obrigatoriedade de investimento de seus excedentes financeiros no desenvolvimento das próprias atividades.

A Tabela 1 apresenta brevemente cada OSS que celebrou parceria com a SMS até agosto de 2015:

A fim de coletar dados sobre as dimensões da capacidade organizacional de ambos os parceiros, e considerando que alguns dos parceiros sem fins lucrativos se comportar como uma "caixa preta" em termos de transparência, utilizamos uma variedade de fontes: pesquisa documental, contratos de parceria e relatórios, curricula vitae de integrantes e diretores, sites de organizações sem fins lucrativos, informações disponíveis no Painel de Gestão da Prefeitura do Rio de Janeiro e entrevistas com o pessoal da SMS.

Os dados foram analisados de acordo com as dimensões de capacidade identificadas na discussão teórica, e a partir disso criamos categorias para cada dimensão de capacidade organizacional, conforme descrito por Fredericksen y London (2000). Tais categorias foram utilizadas como base para uma avaliação global de cada dimensão organizacional, bem como da capacidade como um todo para cada organização sem fins lucrativos e o parceiro público (SMS).

Considerando que as PNPs no setor de saúde têm sido consistentemente o foco de críticas e denúncias, nós nos concentramos no desempenho negativo das parcerias. Os dados relacionados ao desempenho negativo da parceria foram coletados por web scraping de notícias da mídia, além de relatórios das instituições de auditoria que foram analisados para compreender a que tipo de capacidade as dimensões estavam relacionadas. Nos concentramos em: a) reportes da mídia convencional e meios alternativos (blogs e periódicos eletrônicos); b) relatórios da SMS no Painel de Gestão; C) relatório de Auditoria Operacional do Tribunal de Contas da União; e; d) processos e outros documentos do Tribunal de
Justiça do Rio de Janeiro.

As dimensões de capacidade organizacional relacionadas às organizações sem fins lucrativos foram comparadas, construindo 3 (três) tipos de padrões de parcerias: sinergia, quando ambas as dimensões de capacidade do parceiro contribuem para alcançar melhores resultados com as parcerias; complementaridade, quando uma das carências de parceiros em dimensões de capacidade é substituída pela dimensão de forte capacidade da outra parceira, mas não são atingidos resultados mais ambiciosos; e, imposição, quando, no caso de fraca capacidade de um dos parceiros, o parceiro mais forte reforça seu modus operandi nas parcerias.

\section{Descrição e análise dos dados}

\section{Liderança e Visão}

\section{Parceiros sem fins lucrativos}

Analisamos as missões de todas as organizações sem fins lucrativos envolvidas nas parcerias e observamos 4 (quatro) tipos possíveis: a) a missão tem uma orientação explícita relacionada à saúde, demonstrando expertise no setor de saúde; b) a missão enfoca uma orientação contratual, sem foco explícito na saúde; c) a missão se concentra em setores societários alternativos, como caridade ou assistência social, incluindo ou não a saúde; d) não há missão. A Tabela 2 mostra essa tipologia de acordo com a missão OSS.

Ao analisarmos a missão de cada organização, observamos que a maioria das instituições claramente não focaliza suas missões ou conhecimentos em saúde, mas, ao invés disso, são orientadas por um foco na contratualização, destacando seu papel como organização de apoio e gestão em saúde ou alternativa sociais. Alguns dos parceiros sem fins lucrativos têm um propósito claro sobre a saúde, que se traduz também em conhecimentos em saúde como base para a construção de uma parceria com o setor público. Portanto, em todos os casos em que a orientação do setor da saúde não é clara como uma orientação institucional, agravam-se os resultados potenciais das parcerias com o parceiro público, observando uma tendência a perceber tais organizações como meros subcontratados.

A fim de aprofundar a experiência dos parceiros sem fins lucrativos, foram analisados dados relacionados com a experiência dos membros da organização no setor de saúde e trajetória profissional de seus executivos. 
Tabela 1: Organizações Sociais de Saúde (OSS)

\begin{tabular}{|c|c|c|}
\hline OSS & $\begin{array}{l}\text { Ano } \\
\text { de } \\
\text { criação }\end{array}$ & Características \\
\hline SPDM & 1933 & $\begin{array}{l}\text { Associação civil, de natureza filantrópica, reconhecida como entidade de uti- } \\
\text { lidade pública Federal, Estadual e Municipal. Possui um corpo de associados } \\
\text { composto por professores e representantes Associação de Professores, residentes } \\
\text { e estudantes da UNIFESP. }\end{array}$ \\
\hline Fiotec & 1998 & $\begin{array}{l}\text { Fundação de direito privado, sem fins lucrativos, com autonomia administra- } \\
\text { tiva, financeira e patrimônio próprio. Está devidamente credenciada pelo Mi- } \\
\text { nistério da Educação (MEC) e Ministério da Ciência, Tecnologia, Inovações } \\
\text { e Comunicações (MCTIC). Inicialmente, foi criada para gerenciar os projetos } \\
\text { da Escola Nacional de Saúde Pública (Ensp). Com a deliberação da plenária } \\
\text { extraordinária do III Congresso Interno da Fiocruz, ganhou sua atual deno- } \\
\text { minação, assumindo o papel de única fundação de apoio para todo o conjunto } \\
\text { da Fiocruz. }\end{array}$ \\
\hline IABAS & 2008 & $\begin{array}{l}\text { Entidade privada, sem fins lucrativos, qualificada como Organização Social de } \\
\text { Saúde habilitada a atuar na administração de projetos e prestação de serviços } \\
\text { na área da saúde por intermédio de convênios e contratos. }\end{array}$ \\
\hline Viva Rio & 1993 & $\begin{array}{l}\text { Organização sem fins lucrativos que atua na formação de comunidades seguras } \\
\text { e sadias em territórios vulneráveis. Em 2004, chegou ao Haiti a convite da } \\
\text { ONU. Em 2014, instalou-se nos Estados Unidos como Viva Rio Inc. }\end{array}$ \\
\hline CEP 28 & 1967 & $\begin{array}{l}\text { Associação sem fins lucrativos, de direito privado e interesse público, fundada } \\
\text { por profissionais da Santa Casa da Misericórdia do Rio de Janeiro. }\end{array}$ \\
\hline FIBRA & 2000 & $\begin{array}{l}\text { Associação sem fins lucrativos, fundada em Mogi das Cruzes, São Paulo. Seu } \\
\text { principal diretor trabalhou em uma empresa de seguro de saúde e em uma } \\
\text { universidade particular. }\end{array}$ \\
\hline BIOTECH & 2006 & $\begin{array}{l}\text { Uma Organização Não Governamental originada de outra. O prefeito Eduardo } \\
\text { Paes determinou o afastamento imediato dos gestores da Biotech que admi- } \\
\text { nistram os Hospitais Pedro II e o Hospital de Acari. A SMS determinou o } \\
\text { ressarcimento de todo valor que tenha sido desviado nessas parcerias. }\end{array}$ \\
\hline CEJAM & 1991 & $\begin{array}{l}\text { Entidade sem fins lucrativos criada por um grupo de médicos, advogados e } \\
\text { profissionais de saúde do Centro de Referência da Saúde da Mulher e de Nu- } \\
\text { trição Alimentação e Desenvolvimento Infantil - CRSMINADI, Hospital Perola } \\
\text { Byington, para dar apoio àquela Instituição. }\end{array}$ \\
\hline UNIR & 2002 & $\begin{array}{l}\text { Organização Social sem fins lucrativos, criada por um grupo de médicos e profis- } \\
\text { sionais de saúde com autonomia administrativa e financeira, visando principal- } \\
\text { mente a otimização dos recursos, humanização do atendimento e consequente } \\
\text { satisfação do usuário. }\end{array}$ \\
\hline SEB & 1859 & $\begin{array}{l}\text { Associação civil, de natureza filantrópica, fundada por imigrantes espanhis e } \\
\text { possui o Hospital Espanhol do Rio de Janeiro. }\end{array}$ \\
\hline SAS & 2002 & $\begin{array}{l}\text { Organização Social sem fins lucrativos fundada em São Paulo. Opera em vários } \\
\text { setores além da saúde e é qualificada como OSS em vários estados e municípios. }\end{array}$ \\
\hline $\begin{array}{l}\text { Cruz Verme- } \\
\text { lha }\end{array}$ & 1908 & $\begin{array}{l}\text { Sociedade civil filantrópica e sem fins lucrativos que, através de suas filiais } \\
\text { distribuídas em todo território nacional, busca minorar o sofrimento humano } \\
\text { e proporcionar a paz duradoura entre todos os povos. A Filial do Estado } \\
\text { do Rio de Janeiro trouxe para o Estado toda a filosofia desta reconhecida } \\
\text { Sociedade Internacional Socorros, auxiliando os poderes públicos em épocas } \\
\text { de conflitos armados, e através do Comitê Internacional da Cruz Vermelha } \\
\text { faz a fiscalização das Convenções de Genebra e promove a difusão do Direito } \\
\text { Internacional Humanitário. }\end{array}$ \\
\hline GNOSIS & 2009 & $\begin{array}{l}\text { Organização Social sem fins lucrativos fundada para a provisão de serviços de } \\
\text { saúde, inclusive médicos, hospitalares, diagnósticos e serviços de terapia. }\end{array}$ \\
\hline
\end{tabular}


Tabela 2: Liderança e Visão: OSS e Filiação Política

\begin{tabular}{|c|c|c|c|c|}
\hline OSS & Missão & Orientação & $\begin{array}{l}\text { Filiação } \\
\text { Política }\end{array}$ & Valor \\
\hline Fiotec & Prover assistência básica e promoção da saúde. & Foco na saúde & Não & 3 \\
\hline Iabas & $\begin{array}{l}\text { Gerenciar contratos, programas e projetos nos se- } \\
\text { tores de saúde e educação por meio de melhores } \\
\text { práticas, atuando de maneira humada, respeitando } \\
\text { a ética e o comprometimento social. }\end{array}$ & $\begin{array}{l}\text { Foco na contratua- } \\
\text { lização }\end{array}$ & Sim & 2 \\
\hline SPDM & $\begin{array}{l}\text { Promover ações de saúde integradas com o setor } \\
\text { público. }\end{array}$ & Foco na saúde & Sim & 3 \\
\hline Viva Rio & Promover a cultura da paz e a inclusão social. & $\begin{array}{l}\text { Setores sociais al- } \\
\text { ternativos }\end{array}$ & Sim & 1 \\
\hline SEB & Democratizar o acesso à saúde. & Foco na saúde & Não & 3 \\
\hline Cruz Vermelha & $\begin{array}{l}\text { Atuar no caso de guerra. Trabalhar com todos os se- } \\
\text { tores abrangidos na Convenção de Genebra em tem- } \\
\text { pos de paz. }\end{array}$ & $\begin{array}{l}\text { Setores sociais al- } \\
\text { ternativos }\end{array}$ & Não & 1 \\
\hline Cep 28 & $\begin{array}{l}\text { Operar na defesa do meio ambiente, cultura, saúde, } \\
\text { educação e cidadania. }\end{array}$ & $\begin{array}{l}\text { Setores sociais al- } \\
\text { ternativos }\end{array}$ & Não & 1 \\
\hline Gnosis & $\begin{array}{l}\text { Promover o conhecimento nas várias áreas técnicas } \\
\text { e operacionar relacionadas à saúde. }\end{array}$ & Foco na saúde & Não & 3 \\
\hline SAS & $\begin{array}{l}\text { Promover que o Sistema de Saúde trabalhe com } \\
\text { serviços integrados em rede. }\end{array}$ & Foco na saúde & Não & 3 \\
\hline Cejam & $\begin{array}{l}\text { Promover melhor qualidade de vida, oferecendo pro- } \\
\text { gramas nas áreas da saúde, educação e responsabili- } \\
\text { dade social. }\end{array}$ & $\begin{array}{l}\text { Setores sociais al- } \\
\text { ternativos }\end{array}$ & Não & 1 \\
\hline Unir & $\begin{array}{l}\text { Gerenciar processos sociais, aumentando a eficiência } \\
\text { e efetividade, satisfação do cidadão, tendo como base } \\
\text { o desenvolvimento de projetos. }\end{array}$ & $\begin{array}{l}\text { Foco na contratua- } \\
\text { lização }\end{array}$ & Não & 2 \\
\hline Biotech & Desconhecido. & Inexistente & Não & 0 \\
\hline Fibra & Desconhecido. & Inexistente & Não & 0 \\
\hline
\end{tabular}

Tabela 3: Grau de Institucionalização

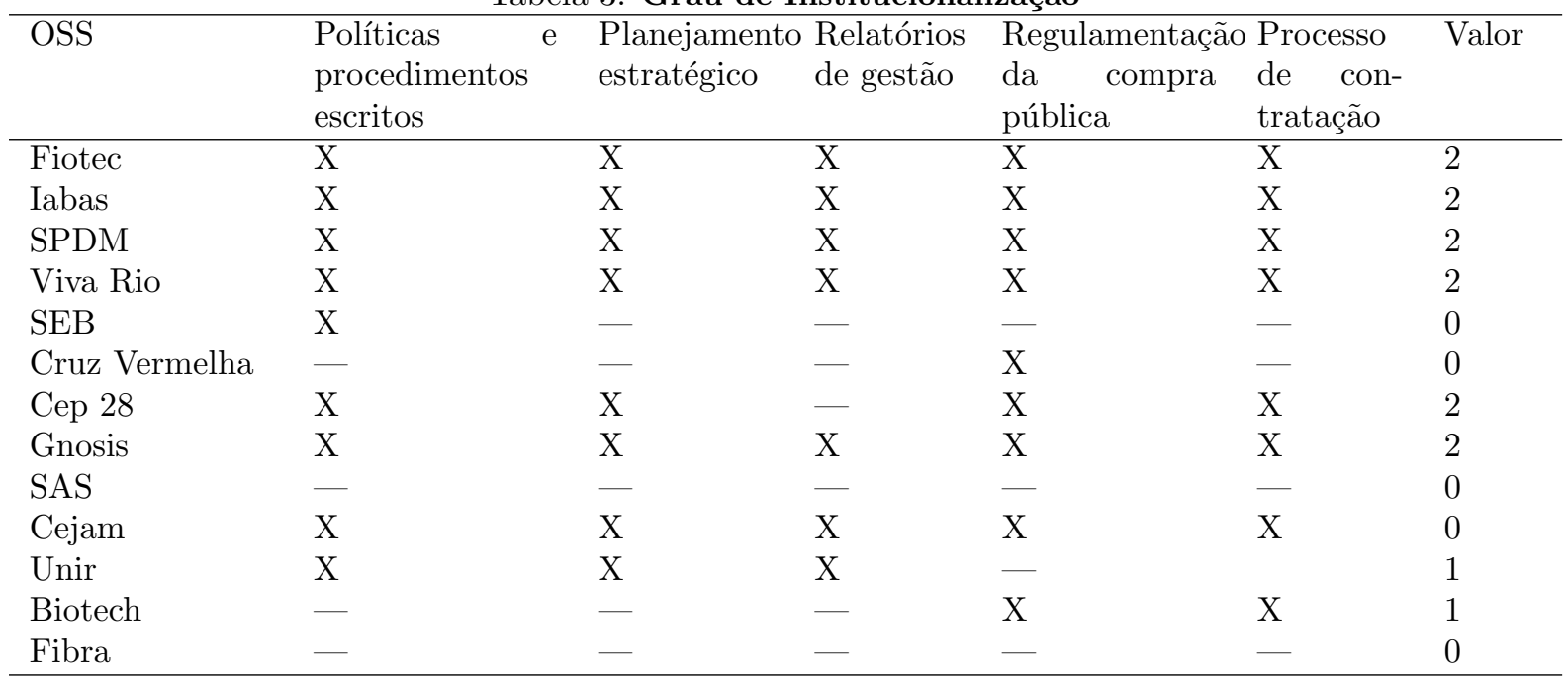

Valores: 5 indicadores significam a disponibilidade das informações consultadas. Assim a pontuação é: a) 5 ou 4 indicadores $=2$; b) 3 ou 2 indicadores $=1$; c) 1 ou 0 indicador $=0$. 
O conselho executivo é um órgão deliberativo, responsável pelo controle do OSS e pela legislação encarregada de descrever os critérios básicos da composição de seus membros, bem como seus principais direitos como requisito para a qualificação da OSS. A Lei $n^{0}$ 5.026/2009 estabelece que a diretoria executiva deve ter notória competência profissional e integridade moral.

Ao analisarmos os conhecimentos dos membros da organização, encontramos membros com expertise na área da saúde em todas as diretorias. Na Fiotec e SPDM, além de profissionais de saúde, todos os membros são servidores públicos: da Fundação Oswaldo Cruz e da Universidade Federal de São Paulo, respectivamente.

De fato, embora pareça suspeito, servidores públicos não são proibidos de fazer parte dos conselhos executivos no Brasil. A limitação ocorre apenas para os servidores públicos nomeados para cargos d Direção e Assessoramento (nomeações políticas). Acreditamos, no entanto, que a possibilidade de servidores públicos ligados às instituições renomadas, bem como a profissionalização de gestores no setor de saúde são bons indicadores para definir o grau de especialização dos líderes dessas instituições.

Além disso, verificamos se algum membro da diretoria executiva está afiliado a um partido político. A IABAS tem a peculiaridade de contar com membros que são parentes de políticos dos partidos PMDB e PSD. Ainda, de acordo com informações eleitorais sobre Luciano Artiori, também componente de seu conselho de administração, ele concorreu para Deputado Federal em 2010, pelo partido DEM. Além disso, vale ressaltar que um dos integrantes do conselho da Viva Rio, Sebastião Correa dos Santos, com o apelido de "Tiao Santos" foi candidado à vereador no Rio de Janeiro, pelo Partido dos Trabalhadores.

\section{Parceiro Público}

Por outro lado, a SMS demonstra alto nível de especialização e orientação de missão para o setor de saúde. É responsável pela formulação e implementação da política de saúde pública a nível municipal, orientada pela missão de "Prover as condições necessárias para promoção, prevenção e assistência em saúde compreendida de forma sistêmica, executando atividades de excelência na área de saúde pública que resultem em melhorias na qualidade de vida da população carioca".

Sua forte orientação e coesão em relação à política de saúde pública é influenciada pela sua participação no Sistema Único de Saúde (SUS). O SUS, criado em 1990, é o sistema de saúde pública brasileiro, baseado em um complexo regime federativo em termos de financiamento e formulação de políticas e implementação. O SUS se refere a uma rede complexa de serviços de saúde prestados por instituições federais, estaduais e municipais. Apesar de suas várias reorganizações, o SUS tem sido responsável pela internalização das principais orientações políticas para a saúde pública no Brasil.

As diretrizes do SUS são incorporadas na visão e ações da SMS: "Assegurar a saúde integral e universal com qualidade a todos os cidadãos do município do Rio de Janeiro, exercendo a função de gestor pleno do Sistema Único de Saúde." A SMS atua com 5 (cinco) Subsecretários, investindo na capacitação e universalização do acesso à saúde pública e na modernização de serviços de saúde mais complexos. Embora o Secretário e Subsecretários sejam politicamente nomeados, eles compartilham de expertise no setor de saúde. Tais características dão à SMS uma liderança coesa que articula os esforços em direção a uma forte orientação na missão de saúde pública.

\section{Grau de institucionalização dos pro- cessos e rotinas organizacionais}

\section{Parceiros sem fins lucrativos}

Analisamos o grau de institucionalização dos procedimentos e rotinas organizacionais de acordo com os dados disponíveis nos sites das OSS, do Painel de Gestão da SMS e nos contratos de parceria, tais como: a) políticas e procedimentos escritos; b) planejamento estratégico; c relatórios de gestão; d) regulamentação da compra pública; e) processo de contratação. A Tabela 3 mostra a presença de indicadores:

Como medida da estabilidade organizacional, observamos que há uma tendência das instituições mais antigas, com um histórico de robusta experiência, têm sua origem ligada a alguns nomes importantes na história da saúde. Nesta lista, podemos incluir alguns dos quais têm mais de quinze anos de criação, especialmente Fiotec, SPDM e a Cruz Vermelha, estando vinculados a instituições reconhecidas nacionalmente como a Escola Nacional de Saúde Pública / Fundação Oswaldo Cruz, Universidade Federal de São Paulo Paulo ou, internacionalmente, ao Movimento Internacional da Cruz Vermelha. No caso das instituições SEB e Fibra, em virtude de seus sites não estarem mais disponíveis durante o período da coleta de dados, não observamos nenhum dos dados de institucio- 
nalização. Em relação a SAS, seu site apresenta apenas um breve comentário sobre sua criação por um grupo de médicos da Universidade Federal de São Paulo, sem detalhes, o que restringiu esse tipo de análise.

\section{Parceiro Público}

Diferentemente de alguns dos parceiros sem fins lucrativos, que parecem ser organizações não estruturadas em termos de procedimentos organizacionais e rotinas, a SMS é uma unidade burocrática típica orientada por uma forte cultura racionallegal (Weber, 1974). Seus processos organizacionais e rotinas são altamente institucionalizados seguindo padrões burocráticos e um alto grau de formalização. Os servidores públicos, os canais formais de promoções, os controles legais e orientados a processos, os manuais de políticas e operacionais padronizados são alguns dos indicadores relacionados ao grau de institucionalização de processos e rotinas organizacionais burocráticos. No entanto, essa cultura burocrática altamente institucionalizada pode ser problemática quando consideramos parcerias, por causa de sua imposição autoritária de seu próprio modus operandi para parceiros sem fins lucrativos.

\section{Principais fontes de financiamento (Planejamento e Práticas Fiscais)}

\section{Parceiros sem fins lucrativos}

Analisamos os indicadores de planejamento fiscal para verificar as fontes primárias de financiamento, buscando se eles têm fontes alternativas de financiamento, e também se trabalham em contratos públicos que não sejam os celebrados com a SMS. Observamos se os seguintes indicadores estavam disponíveis para consulta nos sites de OSS: a) projetos anteriores na área da saúde antes da qualificação na SMS; b) contratos celebrados com outro parceiro público. A Tabela 4 mostra a presença de indicadores:

De acordo com a Lei $\mathrm{n}^{\mathrm{O}} 5.026 / 2009$, a participação em outros projetos de saúde, como parâmetro de avaliação, se deve ao fato de que tais informações são tratadas como alguns dos requisitos específicos para a qualificação como Organização Social de Saúde. Assim, é importante verificar: a) o registro de seu ato constitutivo e; b) o estabelecimento, por pelo menos dois anos, no pleno exercício de atividades no setor saúde.

Analisando as fontes primárias de financiamento, acreditamos que a existência de projetos anteriores na área da saúde e contratos com outros parceiros públicos, mostra que as OSS tendem a ser organizações mais autônomas, uma vez que não são dependentes de um único financiador. Além disso, enfatizamos a importância de a OSS provar sua expertise no setor de saúde, apresentando outros projetos além dos contratados com a SMS, uma vez que deve fornecer feedback para a população.

\section{Parceiro Público}

A SMS é o parceiro responsável pelo financiamento dos contratos de parcerias. Considerando que os parceiros sem fins lucrativos não contribuem financeiramente nos contratos, tal centralização do financiamento também impulsiona uma postura impositiva dos parceiros públicos.

\section{Apoio operacional}

Nos concentramos na dotação de pessoal da OSS como a principal medida de apoio organizacional. É interessante notar que: 1) o Secretário de Saúde exige que toda a contratação de pessoal seja regida pela Consolidação das Leis do Trabalho (CLT); 2) existe a obrigação de padronização dos salários. Isso quer dizer que não há competitividade no mercado de trabalho, já que todos as OSS oferecem os mesmos benefícios financeiros, demonstrando uma clara imposição do parceiro público sobre as organizações sem fins lucrativos.

Foram coletados os relatórios do MHS, disponíveis no Painel de Gerenciamento, a fim de verificar os elementos relacionados às operações do diaa-dia. Foram analisados os parâmetros de relatório estabelecidos pela SMS, tais como: a) absenteísmo; b) turnover dos trabalhadores; c) treinamento medido por hora/homem. A Tabela 5 mostra a presença de indicadores:

Decidimos observar esses indicadores com base nos seguintes constructos: a) absenteísmo: verificar se o OSS possui capacidade operacional em gestão de pessoas; c) turnover/rotatividade de funcionários: verificar se a OSS tem capacidade operacional para contratar profissionais sem desviar a equipe; c) formação medida por hora por homem: verificar se o OSS tem capacidade operacional de qualificar pessoal contratado.

Nossa pesquisa apontou a questão da manutenção dos recursos humanos. Os resultados da UNIR, Gnosis e SPDM confirmam essa idéia. Além disso, há falta de investimento na formação de pessoal contratado, o que pode demonstrar baixa 
Tabela 4: Fontes Primárias de Financiamento

\begin{tabular}{cccc}
\hline OSS & Projetos anteriores na área da Saúde & Contratos com outro Parceiro Público & Valor \\
\hline Fiotec & $\mathrm{X}$ & $\mathrm{X}$ & 2 \\
Iabas & - & - & 0 \\
SPDM & $\mathrm{X}$ & $\mathrm{X}$ & 2 \\
Viva Rio & - & $\mathrm{X}$ & 1 \\
SEB & $\mathrm{X}$ & - & 1 \\
Cruz Vermelha & $\mathrm{X}$ & - & 1 \\
Cep 28 & $\mathrm{X}$ & $\mathrm{X}$ & 2 \\
Gnosis & $\mathrm{X}$ & - & 1 \\
SAS & - & - & 0 \\
Cejam & $\mathrm{X}$ & $\mathrm{X}$ & 2 \\
Unir & - & $\mathrm{X}$ & 1 \\
Biotech & - & - & 0 \\
Fibra & $\mathrm{X}$ & $\mathrm{X}$ & 2 \\
\hline
\end{tabular}

Tabela 5: Apoio Operacional: Recursos Humanos

\begin{tabular}{|c|c|c|c|c|}
\hline Foss & $\begin{array}{l}\text { Absenteísmo (meta } \\
<3 \% \text { ) }\end{array}$ & $\begin{array}{l}\text { Turnover }(\text { meta }< \\
4 \%)\end{array}$ & $\begin{array}{l}\text { Treinamento } \\
>2 h r / h o m)\end{array}$ & Valor \\
\hline Fiotec & $1,24 \%$ & $3,06 \%$ & 1,59 & 2 \\
\hline Iabas & $0,8 \%$ & $0,97 \%$ & 0,72 & 2 \\
\hline SPDM & $1,74 \%$ & $11,61 \%$ & 1,24 & 1 \\
\hline Viva Rio & $1,41 \%$ & $1,33 \%$ & 2,78 & 3 \\
\hline SEB & - & - & 一 & 0 \\
\hline Cruz Vermelha & $0,92 \%$ & $2,51 \%$ & 1,58 & 2 \\
\hline Cep 28 & - & - & - & 0 \\
\hline Gnosis & $2,48 \%$ & $6,06 \%$ & 1,59 & 1 \\
\hline SAS & - & - & - & 0 \\
\hline Cejam & - & - & - & 0 \\
\hline Unir & $2,08 \%$ & $7,34 \%$ & 2,29 & 2 \\
\hline Biotech & - & - & - & 0 \\
\hline Fibra & - & - & - & 0 \\
\hline \multicolumn{5}{|c|}{$\begin{array}{l}\text { Valores: se indicador não estava disponível=0 a) absenteísmo; }<3 \%=1 ;=\text { ou }>3 \%=0 \text { b) turnover; } \\
<4 \%=1 ;=\text { ou }>4 \%=\mathrm{c} \text { ) treinamento medido por hora } / \text { homem } ;>2 h / \text { homem }=1 ;=\text { ou }< \\
2 h / \text { homem }=0\end{array}$} \\
\hline
\end{tabular}


adesão à OSS. Todos esses indicadores parecem constituir um obstáculo à consecução dos objetivos da parceria, ao passo que afetam a qualidade do trabalho e, consequentemente, a qualidade do atendimento ao paciente. Infelizmente, os relatórios de gestão da SEB, SAS, CEJAM, Biotech e Fibra não estavam disponíveis no Painel de Gestão da SMS.

\section{Avaliação Global dos parceiros sem fins lucrativos}

Com base nas notas atribuídas a cada uma das dimensões organizacionais construímos uma avaliação global dos parceiros sem fins lucrativos, como na Tabela 6:

Com base nessa avaliação, criamos uma tipologia de parceiros sem fins lucrativos:

- As organizações sem fins lucrativos "públicas" - Fiotec e SPDM. Tais denominações podem parecer tautológicas, mas representam um tipo muito específico de parceiro sem fins lucrativos, pois são fundações públicas originadas de universidades públicas. Embora essas fundações sejam criadas como organizações sem fins lucrativos, na prática, elas compartilham muito com os parceiros públicos. As fundações públicas são originárias do setor público, têm um foco claro na saúde, compartilham a mesma cultura burocrática com o setor público e contam com funcionários públicos.

- Os "subcontratados" - IABAS, Viva Rio, SAS, CEJAM, Unir, SEB, Gnosis, CEP 28 e Cruz Vermelha. Aqui temos uma série de organizações sem fins lucrativos que não têm uma orientação explícita de missão em saúde, estão focadas na contratação de recursos humanos ou equipamentos para superar as dificuldades operacionais enfrentadas pelos parceiros públicos.

- O "oportunista" - Biotech e Fibra. Essas são organizações oportunistas típicas que não têm nem dados transparentes nem indicadores de capacidade relacionados à parceria. Essas OSS tinham contratos descontinuados por decisão unilateral da SMS, enfrentando até processos de desqualificação. As informações sobre essas organizações e sobre os contratos de gestão que assinaram são muito mais obscuras do que as outras, prejudicando a transparência do modelo.

Nossa pesquisa demonstra que os resultados colaborativos dependem de uma complexa rede de relações que os parceiros desenvolvem ao longo das principais dimensões de capacidade, como demonstrado na Tabela 7.

Observamos três tipos de resultados colaborativos: sinergia, quando ambos os parceiros colaboram para mais objetivos ambiciosos; complementaridade, quando os parceiros sem fins lucrativos substituem a escassez de capacidade do parceiro público (no nosso caso, escassez de parceiros públicos); e imposição, quando, por causa das fraquezas dos parceiros sem fins lucrativos, os parceiros públicos impõem seu próprio modus operandi.

\section{Conclusão}

As PNPs são justificadas devido às várias dificuldades de gestão enfrentadas pelo setor público, além de uma expectativa de que parceiros sem fins lucrativos irão proporcionar melhores resultados na prestação de serviços. Nossa pesquisa revelou que o parceiro sem fins lucrativos enfrenta várias limitações de capacidade organizacional, particularmente relacionadas com as dimensões do apoio organizacional, que obstruem os esforços da SMS para expandir o acesso do cidadão ao serviço de saúde, apesar de ter um forte foco e orientação missionária para a saúde pública. Em outras palavras, as parcerias surgem por necessidade de agentes públicos.

A adequação das dimensões organizacionais específicas de ambos os parceiros influencia os resultados da colaboração. Em nosso caso, percebemos o potencial para uma colaboração sinérgica quando ambos os parceiros partilham conhecimentos e estão fortemente orientados para os valores de saúde pública, como no caso da colaboração com a Fiotec. A complementaridade surge quando os parceiros sem fins lucrativos supriram severas carências gerenciais e organizacionais que os parceiros públicos enfrentam na expansão do acesso e da qualidade dos serviços públicos de saúde, principalmente relacionados à infraestrutura de apoio organizacional (dificuldade de contratação e manutenção de profissionais de saúde, etc). Há um considerável grupo de organizações sem fins lucrativos, cuja orientação da missão assume uma relação contratual com o setor público. Por fim, observamos fortes indicadores de resultados de imposição, quando as organizações sem fins lucrativos não são capazes de institucionalizar seus próprios procedimentos e rotinas organizacionais e dependem exclusivamente de fundos públicos. Na verdade, a maioria dos contratos são detalhados em procedimentos que os parceiros públicos tendem a impor a organizações sem fins lucrativos, como os salários básicos dos profissionais de saúde, o número de computadores e máquinas de impressão que pre- 
Tabela 6: Avaliação Global dos parceiros sem fins lucrativos

\begin{tabular}{|c|c|c|c|c|c|c|}
\hline $\begin{array}{l}\text { Dimensão da capa- } \\
\text { cidade }\end{array}$ & $\begin{array}{l}\text { Liderança } \\
\text { visão }\end{array}$ & $\mathrm{e}$ & Institucionalização & $\begin{array}{l}\text { Fontes de fi- } \\
\text { nanciamento }\end{array}$ & $\begin{array}{l}\text { Apoio opera- } \\
\text { cional }\end{array}$ & Total \\
\hline Fiotec & 3 & & 2 & 2 & 2 & 9 \\
\hline Iabas & 2 & & 2 & 0 & 2 & 6 \\
\hline SPDM & 3 & & 2 & 2 & 1 & 8 \\
\hline Viva Rio & 1 & & 2 & 1 & 3 & 7 \\
\hline SEB & 3 & & 0 & 1 & 0 & 4 \\
\hline Cruz Vermelha & 1 & & 0 & 1 & 2 & 4 \\
\hline Cep 28 & 1 & & 2 & 2 & 0 & 5 \\
\hline Gnosis & 3 & & 2 & 1 & 1 & 7 \\
\hline SAS & 3 & & 0 & 0 & 0 & 3 \\
\hline Cejam & 1 & & 0 & 2 & 0 & 3 \\
\hline Unir & 2 & & 1 & 1 & 2 & 6 \\
\hline Biotech & 0 & & 1 & 0 & 0 & 1 \\
\hline Fibra & 0 & & 0 & 2 & 0 & 2 \\
\hline
\end{tabular}

Conforme "Dimensões da capacidade organizacional que contribuem para as parcerias". Dados da Tabela 2 - Liderança e Visão: OSS e Filiação Política. Dados da Tabela 3 - Grau de Institucionalização. Dados da Tabela 4 - Fontes Primárias de Financiamento. Dados da Tabela 5 - Apoio Operacional: Recursos Humanos. Soma cujo máximo é 10.

Tabela 7: Resultados Colaborativos

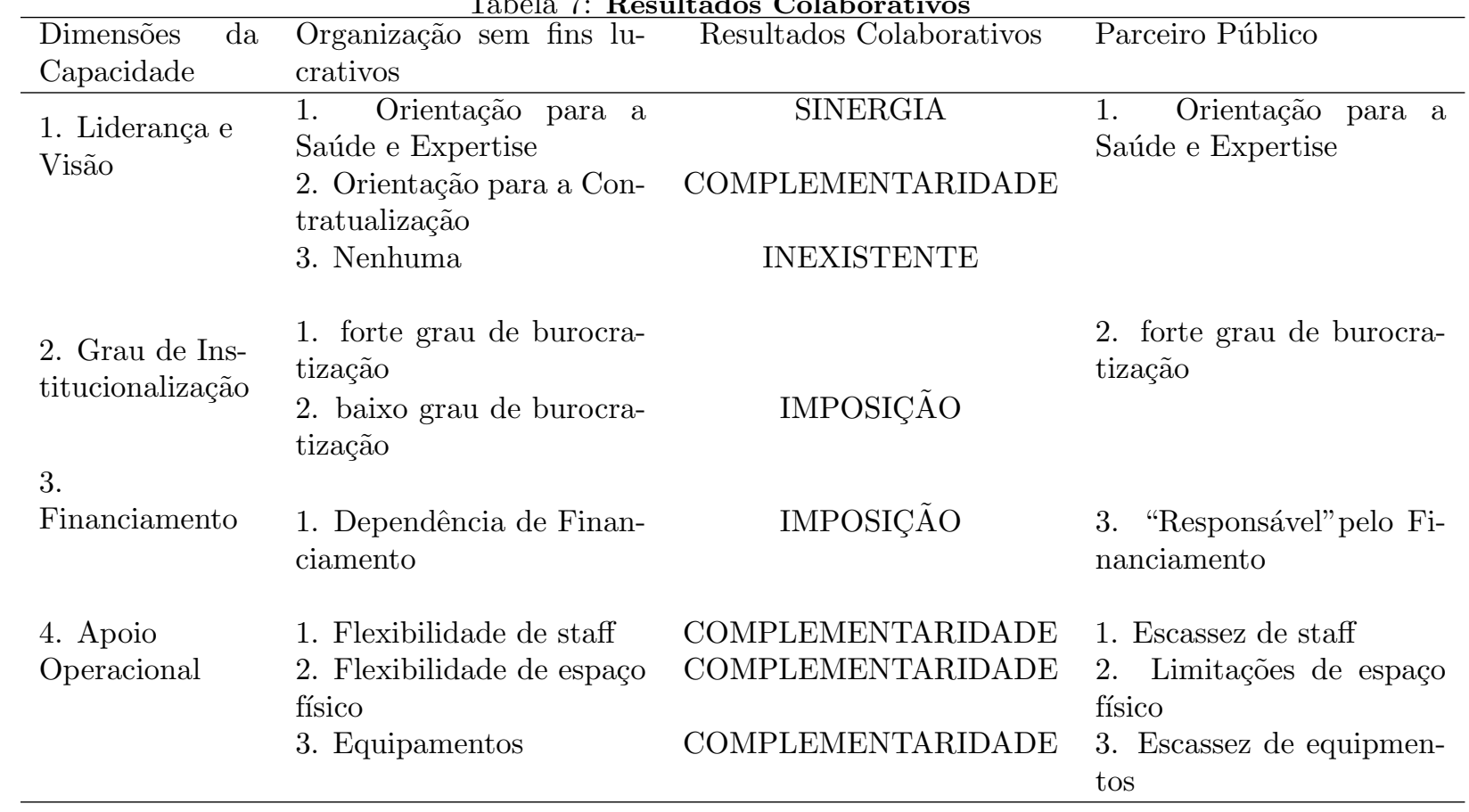


cisam ter, etc.

Nossa pesquisa visou abrir a "caixa preta" das parcerias de saúde no Brasil, que emergem das dimensões de capacidade limitada que o parceiro público enfrenta para cumprir sua missão de universalização e acesso à saúde pública. Apesar dessas nobres intenções, as PNPs enfrentam várias denúncias que indicam uma relação complexa entre parceiros.

Com base na avaliação de várias dimensões de capacidade de parceiros sem fins lucrativos, criamos uma tipologia relacionada a dimensões específicas da capacidade organizacional: as organizações sem fins lucrativos públicas, os subcontratados e os oportunistas.

Ao contrário de outros contextos, as organizações públicas sem fins lucrativos não são um fenômeno tautológico no Brasil, mas referem-se às organizações intimamente relacionadas com o setor público, em termos de orientação de especialistas, base de membros e modus operandi. Essas organizações sem fins lucrativos também foram avaliadas como as organizações mais bem sucedidas em parceria com o setor público. A sua missão e perícia em relação ao parceiro público, mas também a sua partilha do mesmo fundo cultural burocrático facilitam as parcerias e, em algumas das dimensões, tais parceiros sem fins lucrativos podem proporcionar resultados mais sinérgicos das parcerias. Tais fenômenos podem estar relacionados com uma tradição centrada no Estado que prevalece no Brasil, apesar das recentes tentativas de trazer outros atores privados e sem fins lucrativos nos processos de implementação de políticas.

Podemos observar o surgimento de três tipos de resultados colaborativos: sinergia; complementaridade; e imposição. A parte mais problemática da análise refere-se a uma grande parte das parcerias que se baseiam numa relação de complementaridade, onde as organizações sem fins lucrativos são definidas como subcontratos, terceirizando parte das dimensões organizacionais onde o parceiro público enfrenta mais carências e dificuldades (como recursos humanos e outros Dimensões operacionais). Apesar de forçar e impor seu próprio modus operandi em contratos detalhados, os parceiros públicos não são capazes de evitar corrupções e outros tipos de denúncias relacionadas às parcerias. A postura de tal parceiro público não só evita o mau comportamento de organizações sem fins lucrativos, mas também limita a participação de parceiros sem fins lucrativos mais sólidos e relacionados à saúde que evitam a colaboração com o setor público no Brasil.
Como Lasker et al. (2001), também acreditamos que a sinergia é o único resultado possível da colaboração que legitima e sustenta parcerias. Sem a entrega de serviços públicos mais ambiciosos, as parcerias não podem ser sustentadas e continuarão a ser alvo de críticas da opinião pública e de outros agentes públicos. No entanto, os resultados sinérgicos surgem com base em correspondências de dimensões específicas de capacidade, o que requer um forte diagnóstico, competição sem fins lucrativos e soluções ad hoc - ao contrário da abordagem "one fits all" que prevalece no setor público brasileiro.

\section{Referências}

Adams, A. y Deane, J. (2009). Exploring formal and informal dimensions of sports volunteering in England. European sport management quarterly, 9(2):119-140.

Alexander, J. y Nank, R. (2009). Public nonprofit partnership: Realizing the new public service. Administration \& Society, 41(3):364-386.

Alexander, J. A. y Weiner, B. J. (1998). The adoption of the corporate governance model by nonprofit organizations. Nonprofit Management and Leadership, 8(3):223-242.

Alexander, J. A., Weiner, B. J., Metzger, M. E., Shortell, S. M., Bazzoli, G. J., Hasnain-Wynia, R., Sofaer, S., y Conrad, D. A. (2003). Sustainability of collaborative capacity in community health partnerships. Medical Care Research and Review, 60(4_suppl):130S-160S.

Balduck, A.-L., Lucidarme, S., Marlier, M., y Willem, A. (2015). Organizational capacity and organizational ambition in nonprofit and voluntary sports clubs. VOLUNTAS: International Journal of Voluntary and Nonprofit Organizations, 26(5):2023-2043.

Butterfoss, F. D., Goodman, R. M., y Wandersman, A. (1996). Community coalitions for prevention and health promotion: Factors predicting satisfaction, participation, and planning. Health education quarterly, 23(1):65-79.

Cuskelly, G., Evans, G., y Hoye, R. (2004). Problems and issues in the recruitment and retention of sports officials: A report prepared for the Australian Sports Commission. Griffith University, Griffith Business School.

Cuskelly, G., Hoye, R., y Auld, C. (2006). Working with volunteers in sport: Theory and practice. Routledge. 
Eisinger, P. (2002). Organizational capacity and organizational effectiveness among street-level food assistance programs. Nonprofit and Voluntary Sector Quarterly, 31(1):115-130.

Foster-Fishman, P. G., Berkowitz, S. L., Lounsbury, D. W., Jacobson, S., y Allen, N. A. (2001). Building collaborative capacity in community coalitions: A review and integrative framework. American journal of community psychology, 29(2):241-261.

Fredericksen, P. y London, R. (2000). Disconnect in the hollow state: the pivotal role of organizational capacity in community based development organizations. Public Administration Review, 60(3):230-239.

Goodman, R. M., Speers, M. A., McLeroy, K., Fawcett, S., Kegler, M., Parker, E., Smith, S. R., Sterling, T. D., y Wallerstein, N. (1998). Identifying and defining the dimensions of community capacity to provide a basis for measurement. health education \& Behavior, 25(3):258-278.

Hall, M. H., Andrukow, A., Barr, C., Brock, K., de Wit, M., Embuldeniya, D., Jolin, L., Lasby, D., Levesque, B., y Malinsky, E. (2003). The capacity to serve: A qualitative study of the challenges facing Canada's nonprofit and voluntary organizations. Toronto: Canadian Centre for Philanthropy.

Heimovics, R. D., Herman, R. D., y Coughlin, C. L. J. (1993). Executive leadership and resource dependence in nonprofit organizations: A frame analysis. Public Administration Review, pp. 419427.

Henton, D., Melville, J., y Walesh, K. (1997). The age of the civic entrepreneur: restoring civil society and building economic community. National Civic Review, 86(2):149-156.

Huxham, C. y Vangen, S. (2000). Leadership in the shaping and implementation of collaboration agendas: How things happen in a (not quite) joined-up world. Academy of Management journal, 43(6):1159-1175.

Kanter, R. M. (1994). Collaborative advantage. Harvard business review, 72(4):96-108.

Kim, M., Chelladurai, P., y Trail, G. T. (2007). A model of volunteer retention in youth sport. Journal of Sport Management, 21(2):151-171.

Lambright, K. T., Mischen, P. A., y Laramee, C. B. (2010). Building trust in public and nonprofit networks: personal, dyadic, and third-party influences. The American Review of Public Administration, 40(1):64-82.
Lasker, R. D. (1997). Medicine and public health: the power of collaboration.

Lasker, R. D., Weiss, E. S., y Miller, R. (2001). Partnership synergy: a practical framework for studying and strengthening the collaborative advantage. The Milbank Quarterly, 79(2):179-205.

Lecy, J. D. y Van Slyke, D. M. (2013). Nonprofit sector growth and density: Testing theories of government support. Journal of Public Administration Research and Theory, 23(1):189-214.

Lodge, M. y Wegrich, K. (2014). The problemsolving capacity of the modern state: governance challenges and administrative capacities. Oxford University Press, USA.

Mayo, M. (1997). Partnerships for regeneration and community development: some opportunities, challenges and constraints. Critical Social Policy, 17(52):3-26.

Mcphee, P. y Bare, J. (2001). Building Capacity in Nonprofit Organizations. The Urban Institute.

Millar, P. y Doherty, A. (2016). Capacity building in nonprofit sport organizations: Development of a process model. Sport management review, 19(4):365-377.

Misener, K. y Doherty, A. (2009). A case study of organizational capacity in nonprofit community sport. Journal of sport management, 23(4):457482 .

Paynter, S. y Berner, M. (2014). Organizational capacity of nonprofit social service agencies. Journal of health and human services administration, pp. 111-145.

Poncelet, J.-L. y de Ville, d. G. C. (1995). Disaster preparedness: institutional capacity building in the Americas. World health statistics quarterly. Rapport trimestriel de statistiques sanitaires mondiales, 49(3-4):195-199.

Rainey, H. G. y Steinbauer, P. (1999). Galloping elephants: Developing elements of a theory of effective government organizations. Journal of public administration research and theory, 9(1):132 .

Reich, M. R. (2002). Public-private partnerships for public health. Public-private partnerships for public health, pp. 1-18.

Schuh, R. G. y Leviton, L. C. (2006). A framework to assess the development and capacity of nonprofit agencies. Evaluation and Program Planning, 29(2):171-179. 
Seippel, Ø. (2002). Volunteers and professionals in Norwegian sport organizations. Voluntas: international journal of voluntary and nonprofit organizations, 13(3):253-270.

Sharpe, E. K. (2006). Resources at the grassroots of recreation: Organizational capacity and quality of experience in a community sport organization. Leisure sciences, 28(4):385-401.

Skelcher, C. (2005). Public-private partnerships. Oxford University Press, New York.

Sowa, J. E., Selden, S. C., y Sandfort, J. R. (2004). No longer unmeasurable? A multidimensional integrated model of nonprofit organizational effectiveness. Nonprofit and voluntary sector quarterly, 33(4):711-728.

Vinzant, D. H. y Vinzant, J. C. (1996). Strategy and organizational capacity: Finding a fit. Public Productivity \& Management Review, pp. 139-157.

Vos, S., Breesch, D., Késenne, S., Lagae, W., Van Hoecke, J., Vanreusel, B., y Scheerder, J. (2012). The value of human resources in nonpublic sports providers: the importance of volunteers in non-profit sports clubs versus professionals in for-profit fitness and health clubs. International Journal of Sport Management and Marketing 2, 11(1-2):3-25.

Walker, C. y Weinheimer, M. (1998). Community Development.

Warner, S., Newland, B. L., y Green, B. C. (2011). More than motivation: Reconsidering volunteer management tools. Journal of Sport Management, 25(5):391-407.

Wicker, P. y Breuer, C. (2011). Scarcity of resources in German non-profit sport clubs. Sport management review, 14(2):188-201.

Zucker, L. G. (1987). Institutional theories of organization. Annual review of sociology, 13(1):443464. 